\title{
Dermatose bulleuse hémorragique probablement associée au fondaparinux
}

\author{
Catherine Anne Lovatt MD, Wen Qing Wendy Ye MD MSc, Haroon Yousuf MD MScS
}

citation : CMAJ 2021 May 31;193:E801-4. doi : 10.1503/cmaj.202747-f

Voir la version anglaise de l'article ici : www.cmaj.ca/lookup/doi/10.1503/cmaj.202747

$\mathbf{U}$ ne femme de 90 ans a été admise dans un hôpital de soins tertiaires pour une hypoxie secondaire à un œdème pulmonaire; elle recevait une antibiothérapie pour traiter une infection de sa prothèse de hanche. Les antécédents médicaux de la patiente comprenaient une thrombocytopénie induite par la daltéparine, une fibrillation auriculaire (score $\mathrm{CHADS}_{2}$ de 3 ) et une hémorragie digestive basse liée à une maladie diverticulaire. La patiente n'a signalé aucune allergie cutanée. Quatre semaines avant l'admission, elle avait subi le retrait d'une prothèse de hanche droite, ainsi qu'un débridement et une révision sous la forme d'une hémiarthroplastie à fixation cimentée de la hanche droite en raison d'une infection à Staphylococcus aureus sensible à la méthicilline. Au moment de l'admission, elle suivait un traitement de 6 semaines à la céfalexine et à la rifampicine. Elle avait déjà pris de l'apixaban pour la prévention d'un AVC, mais, avant son admission, l'apixaban avait été remplacé par la warfarine en raison de son interaction avec la rifampicine. Toutefois, étant donné l'interaction entre la warfarine et la rifampicine et ses antécédents complexes de thrombose, nous avons consulté le service traitant les thromboses pour la gestion du traitement anticoagulant de la patiente. Nous avons alors commencé des injections quotidiennes de fondaparinux dans l'abdomen tout en poursuivant l'administration de rifampicine. Le furosémide par voie intraveineuse a permis d'éliminer la dyspnée de la patiente.

Au quatrième jour du traitement au fondaparinux, la patiente a développé des lésions hémorragiques d'une intense coloration pourpre, non associées à un traumatisme et ni douloureuses ni prurigineuses, et des phlyctènes sur l'avant-bras gauche (figure 1). Au cours des 2 journées suivantes, ces lésions ont progressé en taille et en nombre pour finalement atteindre son bras droit, mais sont restées cantonnées aux extrémités supérieures. Les muqueuses n'ont pas été touchées. Les phlyctènes épicondyliennes gauches se sont rompues au sixième jour de traitement par fondaparinux, entraînant une hémorragie importante. Le taux d'hémoglobine de la patiente est passé de $91 \mathrm{~g} / \mathrm{L}$ (taux normal 130-180) au jour 4 du traite-

\section{Points clés}

- La dermatose bulleuse hémorragique est une réaction indésirable, non immunitaire et peu fréquente aux anticoagulants à base d'héparine, notamment l'héparine non fractionnée, l'héparine de faible poids moléculaire et le fondaparinux. Elle survient généralement dans les 7 jours suivant l'exposition au médicament.

- La réaction s'observe le plus fréquemment chez les adultes âgés, et les lésions se situent généralement sur les membres.

- Les résultats aux tests de coagulation tels que le rapport international normalisé, le temps de thromboplastine partielle activée ou le temps de prothrombine ne changent généralement pas.

- La dermatose bulleuse hémorragique se résorbe d'elle-même. Toutefois, une surveillance attentive des saignements et une prise en charge adaptée, comme le soin des plaies et la transfusion de produits sanguins, peuvent être nécessaires.

ment par fondaparinux à $70 \mathrm{~g} / \mathrm{L}$ au jour 6 , mais ses paramètres de coagulation se situaient dans la tranche supérieure de la plage normale : rapport international normalisé (RIN) de 1,2 (plage normale $0,8-1,2$ ), temps de prothrombine à 14,9 secondes (plage normale 11-15 secondes) et temps de thromboplastine partielle activée à 41 secondes (plage normale 22-35 secondes). Nous n'avons pas détecté d'autres sources de saignement ou d'hémolyse. Nous avons administré à la patiente 4 transfusions de concentré de globules rouges sur 3 jours, et avons arrêté le fondaparinux le $7^{\mathrm{e}}$ jour en raison de l'hémorragie en cours. Ses lésions cutanées ont été recouvertes de pansements de compression imbibés d'acide tranexamique. Une revue approfondie des médicaments n'a pas révélé d'autres causes potentielles pour les phlyctènes hémorragiques.

Les analyses de rhumatologie au jour 6 ont donné des résultats positifs pour les anticorps extractibles et les anticorps anti-Ro/SSA 52 à 4,1 (plage normale $<1,0$ ). Nous avons soupçonné des phlyctènes hémorragiques liées à une vascularite, mais une biopsie à l'emporte-pièce du bras gauche réalisée le $10^{\mathrm{e}}$ jour n'a montré qu'une élastose solaire et une 

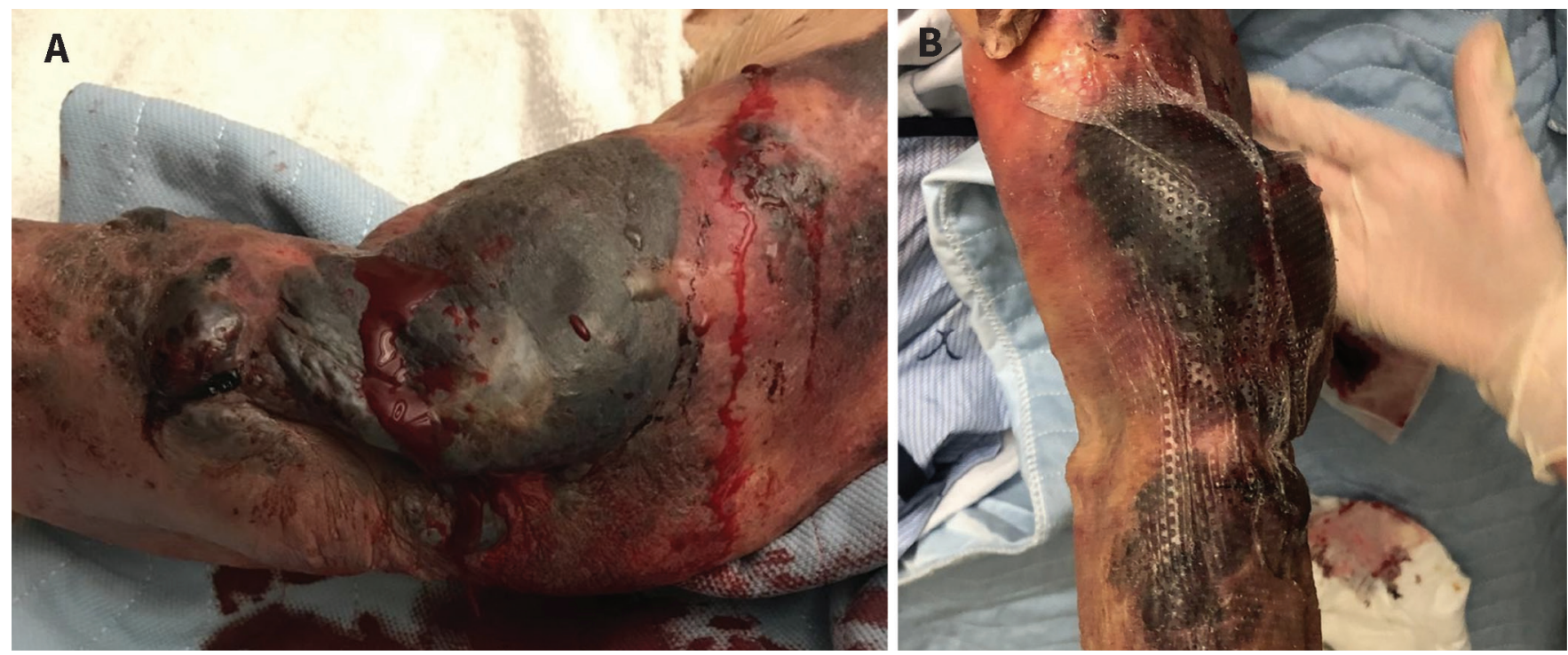

Figure 1 : Photographies des lésions dues à la dermatose bulleuse hémorragique sur le bras et la main gauche d'une femme de 90 ans, au jour 6 ( $A$ ) et au jour 10 (B) de l'administration du fondaparinux.
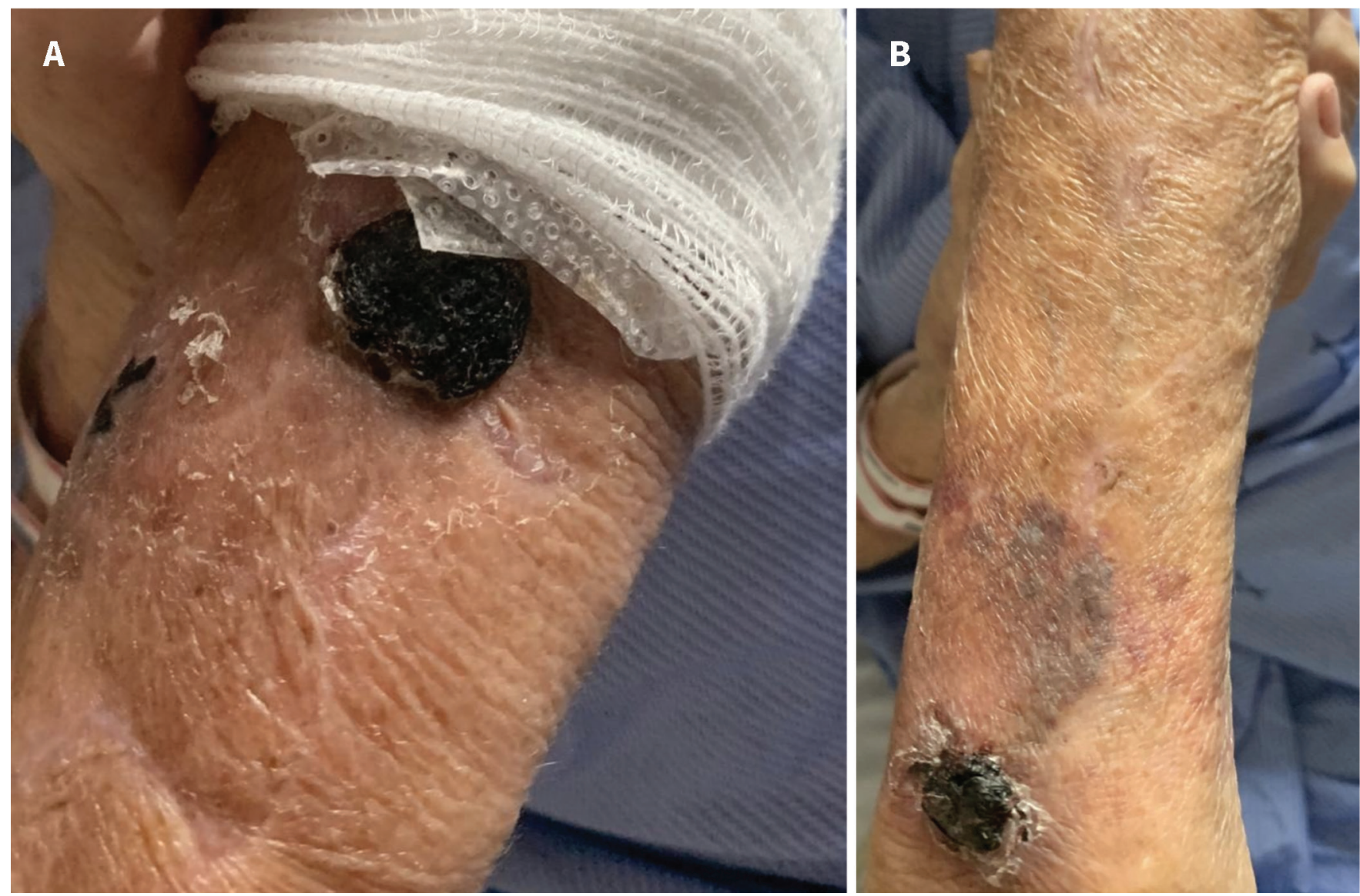

Figure 2 : Résorption de la dermatose bulleuse hémorragique sur le bras gauche de la patiente au jour 23.

atrophie de l'épiderme, incompatibles avec une vascularite. En consultation avec le service de rhumatologie, nous avons établi que le résultat de l'analyse des anticorps extractibles était probablement erroné.

Nous avons diagnostiqué une dermatose bulleuse hémorragique induite par le fondaparinux. Nous avons clairement con- signé les réactions potentiellement mortelles à tous les produits liés à l'héparine dans le dossier médical, et en avons discuté avec la patiente et sa famille.

Sur les conseils de l'équipe des maladies infectieuses, nous avons arrêté la rifampicine et remis la patiente sous apixaban à faible dose pour la prévention en continu d'AVC. Les phlyctènes 
hémorragiques se sont complètement résorbées après l'arrêt du fondaparinux; leur dessiccation et l'involution de la décoloration cutanée sont survenues au $23^{\mathrm{e}}$ jour (figure 2).

Cette admission à l'hôpital a eu une profonde incidence sur la qualité de vie de la patiente. En raison du déconditionnement et de la fragilité qui en a résulté, elle a finalement demandé à être placée dans un centre de soins de longue durée.

\section{Discussion}

\section{Épidémiologie et diagnostic différentiel}

La dermatose bulleuse hémorragique est une réaction systémique indésirable rare, à médiation non immunitaire, liée aux anticoagulants à base d'héparine. Elle a été surtout signalée avec l'énoxaparine et survient habituellement après 7 jours (intervalle 3-270 jours) d'exposition au médicament ${ }^{1,2}$. Cette affection fait probablement l'objet d'une sousdéclaration; d'ailleurs, les articles revus par les pairs mentionnent seulement 97 cas $^{1,3-6}$. La dermatose bulleuse hémorragique a été signalée précédemment en relation avec l'association fondaparinux-warfarine $\mathrm{e}^{7,8}$. Aucun cas associé à des anticoagulants oraux directs n'a été rapporté. Dans leur résumé de 91 cas rapportés de dermatose bulleuse hémorragique, Russo et ses collègues ont constaté que les lésions n'étaient habituellement pas douloureuses, prurigineuses ou associées à d'autres symptômes systémiques. La distribution des lésions s'observait le plus souvent loin des sites d'injection, les principales zones touchées étant les extrémités, suivies du torse. Les muqueuses étaient rarement atteintes ${ }^{1,4}$. Dans la plupart des cas, cette affection disparaissait d'elle-même, dans un délai moyen de 13 jours ${ }^{9}$. Quatre décès y ont été associés ${ }^{1}$.

La gamme des diagnostics différentiels des phlyctènes hémorragiques est vaste (tableau 1). Pour notre patiente, le diagnostic différentiel comprenait la nécrose cutanée induite par la warfarine, les phlyctènes hémorragiques liées à une vascularite, la pemphigoïde bulleuse induite par la céfalexine, les ampoules de friction mécanique, l'hémophilie acquise et la cellulite bulleuse hémorragique. La nécrose cutanée induite par la warfarine survient généralement quelques jours après l'exposition au médicament et se développe dans les tissus adipeux sous-cutanés, notamment ceux des seins et des cuisses. Cette description ne correspondait pas à l'apparition ou à la distribution des lésions chez notre patiente. La pemphigoïde bulleuse et les nombreuses formes de réactions cutanées à médiation médicamenteuse constituent des réponses immunitaires associées à une infiltration d'éosinophiles, ce que ne reflétait pas la biopsie cutanée de la patiente $\mathrm{e}^{7,10}$.

Les phlyctènes hémorragiques associées à une hémophilie acquise (p. ex., en cas de déficit acquis en facteur VIII) sont également liées au système immunitaire et devraient modifier les paramètres d'anticoagulation, comme le rapport international normalisé ou le temps de thromboplastine partielle activée ${ }^{11}$. Aucun signe d'infection cutanée ou systémique

\section{Tableau 1 : Diagnostic différentiel des phlyctènes} hémorragiques, ${ }^{5,10}$

Médiation immunitaire

Vascularite leucocytoclasique

Pemphigoïde bulleuse atypique

Pemphigoïde bulleuse d'origine médicamenteuse

Épidermolyse bulleuse acquise

Pemphigus vulgaire

Pemphigus paranéoplasique

Pyoderma gangrenosum bulleux

Hématogène

Purpura de Henoch-Schönlein

Porphyrie cutanée tardive

Médiation

médicamenteuse ou

toxinique

Hémophilie

Nécrose cutanée induite par l'héparine

Nécrose induite par la warfarine

Infectieuse Morsure d'araignée hémorragique

Fasciite nécrosante

Mécanique

Cellulite bulleuse hémorragique

Virus varicelle-zona

Cloques de sang dues à la friction

n'a été détecté. Compte tenu du rapport de pathologie pour la biopsie à l'emporte-pièce, de la corrélation temporelle avec l'initiation du traitement par fondaparinux, de la résorption spontanée des lésions et de l'absence d'autres explications plausibles, nous avons diagnostiqué un cas probable de dermatose bulleuse hémorragique induite par le fondaparinux selon le système d'évaluation de la causalité du Centre de pharmacovigilance d'Uppsala, un centre collaborateur de l’Organisation mondiale de la Santé12.

\section{Profil pathophysiologique}

La pathophysiologie de la dermatose bulleuse hémorragique n'a pas été totalement élucidée. Les biopsies cutanées semblent indiquer une hémorragie intraépidermique en l'absence de cellules immunitaires ou de marqueurs d'inflammation ${ }^{1}$. En raison de l'âge moyen avancé des patients atteints de dermatose bulleuse hémorragique, certains ont émis l'hypothèse d'un lien causal avec la fragilité des structures épidermiques et dermiques. Cette hypothèse cadrerait avec le cas de notre patiente, compte tenu de l'atrophie de l'épiderme décrite dans la biopsie cutanée. La patiente présentait une peau crêpée due à son âge avancé, mais aucune lésion ou rupture cutanée n'avait été remarquée auparavant.

La patiente a pu présenter une prédisposition à la dermatose bulleuse hémorragique, en raison de la combinaison d'un risque accru de nécrose cutanée attribuable à la thrombocytopénie induite par l'héparine 6 mois auparavant, d'un retard de cicatrisation et d'une fragilité cutanée accrue due à son âge et à ses comorbidités ${ }^{1}$. La thrombocytopénie induite par l'héparine est une réaction indésirable aux produits à base d'héparine médiée par l'immunoglobuline $\mathrm{G}^{13}$. Des 
études de cas ont décrit une association possible entre la dermatose bulleuse hémorragique et les réactions eczémateuses aux sites d'injection, laissant penser à une réaction d'hypersensibilité de type IV ${ }^{1}$. Il s'agit d'un mécanisme immunitaire qui est différent de celui intervenant dans la thrombocytopénie induite par l'héparine et qui ne confère pas une sensibilité à d'autres réactions immunitaires. On n'a signalé aucune augmentation des thromboembolies veineuses ou artérielles chez les patients atteints de dermatose bulleuse hémorragique, alors que c'est un élément essentiel du diagnostic de la thrombocytopénie induite par l'héparine. Étant donné que la dermatose bulleuse hémorragique n'est pas d'origine immunitaire, les antécédents de thrombocytopénie induite par l'héparine de la patiente et le développement ultérieur de la dermatose bulleuse hémorragique lors de la prise de fondaparinux relèvent probablement d'une coïncidence.

\section{Prise en charge}

L'arrêt de l'agent incriminé ou le passage à un autre agent anticoagulant constituent les stratégies thérapeutiques les plus courantes, et on y a eu recours dans environ $60 \%$ des cas recensés ${ }^{1}$. Comme la dermatose bulleuse hémorragique a tendance à se résorber d'elle-même, certains spécialistes font remarquer que l'arrêt de l'agent incriminé ne change pas grand chose dans le délai de guérison. Environ $23 \%$ des cas se sont résorbés malgré la poursuite de la prise du médicament soupçonné d'être à l'origine de l'affection, et 14,3\% des cas se sont résolus après une diminution de la dose ou un changement d'anticoagulant ${ }^{1}$. Au vu de la gravité du cas de la patiente, qui présentait une instabilité hémodynamique nécessitant une transfusion, nous avons jugé prudent d'interrompre tous les agents anticoagulants. Nous avons signalé ce cas au Programme Canada Vigilance.

\section{Conclusion}

La dermatose bulleuse hémorragique est une réaction médicamenteuse rare sans médiation immunologique qui disparaît d'elle-même. Elle survient en cas de prise d'anticoagulants à base d'héparine. Cette réaction, plus fréquente chez les adultes âgés, risque d'entraîner une instabilité hémodynamique requérant une transfusion. Les risques et les bénéfices d'un traitement anticoagulant continu doivent donc être évalués au cas par cas.

\section{Références}

1. Russo A, Curtis S, Balbuena-Merle R, et al. Bullous hemorrhagic dermatosis is an under-recognized side effect of full dose low-molecular weight heparin: a case report and review of the literature. Exp Hematol Oncol 2018;7:15.

2. Roy SF, Watson P, Bouffard D. Bullous hemorrhagic dermatosis. CMAJ 2020;192:E12.

3. Harris HB, Kurth BJ, Lam TK, et al. Heparin-induced bullous hemorrhagic dermatosis confined to the oral mucosa. Cutis 2019;103:365, 366, 370.

4. Snow SC, Pearson DR, Fathi R, et al. Heparin-induced haemorrhagic bullous dermatosis. Clin Exp Dermatol 2018;43:393-8.

5. Gérard A, Levavasseur M, Gaboriau L, et al. Hemorrhagic bullous dermatosis (HBD): a rare side-effect of heparins [article en français]. Ann Dermatol Venereol 2020;147:446-50.
6. Maldonado Cid P, Moreno Alonso de Celada R, Herranz Pinto P, et al. Bullous hemorrhagic dermatosis at sites distant from subcutaneous injections of heparin: a report of 5 cases. J Am Acad Dermatol 2012;67:e220-2.

7. Komforti MK, Bressler ES, Selim MA, et al. A rare cutaneous manifestation of hemorrhagic bullae to low-molecular-weight heparin and fondaparinux: report of two cases. J Cutan Pathol 2017;44:104-6.

8. Ferguson A, Golden S. Hemorrhagic bullous dermatosis caused by warfarin therapy. JAAD Case Rep 2016;2:156-8.

9. Dejuán-Bitriá C, Estébanez M, López-Galán C, et al. Hemorrhagic bullous dermatosis after bilateral radial catheterization. Coron Artery Dis 2018; 29:694-5.

10. Schmidt E, Della Torre R, Borradori L. Clinical features and practical diagnosis of bullous pemphigoid. Immunol Allergy Clin North Am 2012;32:217-32.

11. Binet Q, Lambert C, Sacré L, et al. Successful management of acquired hemophilia a associated with bullous pemphigoid: a case report and review of the literature. Case Rep Hematol 2017;2017. doi: 10.1155/2017/2057019.

12. The use of the WHO-UMC system for standardised case causality assessment. Uppsala (Sweden): Uppsala Monitoring Centre; 2018. Accessible ici : www. WHO-UMC.org/graphics/4409.pdf (consulté le 16 févr. 2021).

13. Godet $\mathrm{T}$, Perbet S, Lebreton A, et al. Low molecular weight heparin induced skin necrosis without platelet fall revealing immunoallergic heparin induced thrombocytopenia. Case Rep Hematol 2013;2013. doi: $10.1155 / 2013 / 849168$.

Intérêts concurrents : Aucun déclaré.

Cet article a été révisé par des pairs.

Les auteurs ont obtenu le consentement de la patiente.

Affiliations : Département de médecine (Lovatt, Ye, Yousuf), Faculté des sciences de la santé, Université McMaster; Hamilton Health Sciences (Yousuf), Hamilton, Ont.

Collaborateurs : Tous les auteurs ont contribué à la conception du travail, ont rédigé le manuscrit et en ont révisé de façon critique le contenu intellectuel important; ils ont donné leur approbation finale pour la version destinée à être publiée et assument l'entière responsabilité de tous les aspects du travail.

Propriété intellectuelle du contenu : Il s'agit d'un article en libre accès distribué conformément aux modalités de la licence Creative Commons Attribution (CC BY-NC-ND 4.0), qui permet l'utilisation, la diffusion et la reproduction dans tout médium à la condition que la publication originale soit adéquatement citée, que l'utilisation se fasse à des fins non commerciales (c.-à-d., recherche ou éducation) et qu'aucune modification ni adaptation n'y soit apportée. Voir : https://creativecommons.org/licenses/ by-nc-nd/4.0/deed.fr.

Correspondance : Haroon Yousuf, yousufhs@mcmaster.ca

La section Études de cas présente de brefs rapports de cas à partir desquels des leçons claires et pratiques peuvent être tirées. Les rapports portant sur des cas typiques de problèmes importants, mais rares ou sur des cas atypiques importants de problèmes courants sont privilégiés. Chaque article commence par la présentation du cas (500 mots maximum), laquelle est suivie d'une discussion sur l'affection sous-jacente (1000 mots maximum). La soumission d'éléments visuels (p. ex., tableaux des diagnostics différentiels, des caractéristiques cliniques ou de la méthode diagnostique) est encouragée. Le consentement des patients doit impérativement être obtenu pour la publication de leur cas. Renseignements destinés aux auteurs : www.cmaj.ca. 